Open Access

\title{
Sepsis modeling in mice: ligation length is a major severity factor in cecal ligation and puncture
}

Stéphanie Ruiz ${ }^{1,2^{*}}$ (D) Fanny Vardon-Bounes ${ }^{1,2}$, Virginie Merlet-Dupuy ${ }^{1}$, Jean-Marie Conil ${ }^{1}$, Marie Buléon ${ }^{4}$, Olivier Fourcade ${ }^{1,3}$, Ivan Tack ${ }^{4,5}$ and Vincent Minville $e^{1,2}$

\author{
* Correspondence: \\ ruiz.stephanie@chu-toulouse.fr \\ 'Department of Anesthesiology and \\ Intensive Care, Rangueil Hospital, \\ University Hospital of Toulouse, 1 \\ Avenue du Professeur Jean Poulhès \\ TSA 50032, 31059 Toulouse, Cedex \\ 9, France \\ ${ }^{2}$ Inserm/UPS UMR 1048 - I2MC, \\ Equipe 3, Toulouse, France \\ Full list of author information is \\ available at the end of the article
}

\begin{abstract}
Background: The cecal ligation and puncture (CLP) model, a gold standard in sepsis research, is associated with an important variability in mortality. While the number of punctures and needle size is well described in CLP animal studies, the length of cecal ligation is often not. The relationship between cecal ligation and survival in mice is briefly reported in the literature; therefore, we devised an investigation in mice of the consequences of three standardized cecal ligation lengths on mortality and the severity of the ensued sepsis.
\end{abstract}

Methods: Male C57BL/6J mice underwent standardized CLP. The cecum was ligated at 5,20 , or $100 \%$ of its total length and further perforated by a single $20-G$ puncture. Mortality was analyzed. We assessed blood lactate, serum creatinine levels, and serum cytokines (TNF-a, IL-1 $\beta, I L-6$, and IL-10) after procedure in a control group and in ligated mice.

Results: Mortality was directly related to ligation length: median survival was $24 \mathrm{~h}$ for the "100 \%" group and $44 \mathrm{~h}$ for the "20 \%" group. Blood lactate increased proportionally with the ligation length. At 6 h post-procedure, pro-inflammatory cytokines significantly increased in the ligated group with significantly higher serum levels of IL-6 in the $100 \%$ group compared to the other ligated groups. The $20 \%$ group exhibited the characteristics of septic shock with hypotension below $65 \mathrm{mmHg}$, pro-inflammatory balance, organ dysfunction, and hyperlactatemia.

Conclusions: Cecal ligation length appears to be a major limiting factor in the mouse CLP model. Thus, this experimental model should be performed with high consistency in future protocol designs.

Keywords: Cecal ligation and puncture, Mice, Cytokine, Sepsis

\section{Background}

Severe sepsis results from a complex and dynamic pathophysiology; therefore, a better understanding of the inflammatory process leading to sepsis is essential [1]. Although they do not reflect entirely the clinical complexity, animal models remain a valuable approach to developing new therapeutic strategies. Various animal models of sepsis have already been developed such as intravascular infusion of endotoxin (lipopolysaccharide (LPS)), live bacteria or viruses, bacterial peritonitis, cecal ligation and puncture (CLP), soft tissue infection, pneumonia model, and meningitidis model [2-6]. However,

(c) 2016 The Author(s). Open Access This article is distributed under the terms of the Creative Commons Attribution 4.0 International License (http://creativecommons.org/licenses/by/4.0/), which permits unrestricted use, distribution, and reproduction in any medium, provided you give appropriate credit to the original author(s) and the source, provide a link to the Creative Commons license, and indicate if changes were made. 
since 1998 Deitch pointed out that an important number of failures in new therapeutic approaches may be due to the use of inappropriate experimental models [7]. An endotoxic model (LPS injection) mimics poisoning more than infection. In the LPS endotoxic model, the cytokines peak early and transiently, whereas in the CLP model, the pro-inflammatory response is delayed and persists over time [8]. LPS model mortality occurs early, most likely due to the effects of the intense inflammatory response on the cardiovascular system, whereas in the CLP model, mortality is delayed with multiple organ failure complicating induced peritonitis. In humans, endotoxic shocks are rare and sepsis origin is often localized. The CLP model is the most widely used model for experimental sepsis and is currently considered as a gold standard in research since it mimics the nature and evolution of severe sepsis in humans [5, 9]. Ensuing a simple procedure, the model induces sepsis secondary to a stercoral peritonitis, followed by a polymicrobian translocation in the blood circulation with an early inflammatory phase, after which an anti-inflammatory response develops [2]. However, significant variability on mortality from one experimental protocol to another can lead to differing interpretations of the results. That being said, survival rates can vary from 20 to $50 \%$. The main determinants of mortality are the size of the needle used for cecal puncture; the number of punctures, generally between 1 and 4; and the use of antibiotics and/or fluid resuscitation [10, 11]. While the number of punctures and needle size is standardized, the length of cecal ligation is often not described in CLP animal studies.

To the best of our knowledge, only few brief descriptions exist regarding cecal ligation and survival in the mouse CLP model [10, 12]. We investigated in nonresuscitated $\mathrm{C} 57 \mathrm{BL} / 6 \mathrm{~J}$ male mice the consequences of several standardized distances of CLP on mortality and sepsis severity. To do so, we used organ failure markers such as serum creatinine levels (as an early sign of acute kidney injury), serum lactate, and the kinetics of the inflammatory state reflected by cytokine synthesis including TNF- $\alpha$, IL-1 $\beta$, IL-6, and IL-10.

\section{Methods}

\section{Animals}

C57BL/6J wild-type mice were obtained from Harlan (Harlan France, Gannat, France). We used male animals aged 20 weeks, weighing 25-30 g. Animal experimentation was performed according to national and institutional animal care and ethical guidelines and was approved by the local board. Mice were housed in a temperature-controlled room on a 12-h night-dark cycle. Four animals were placed in a cage and had access to water and food ad libitum. The mice were not fasted prior to CLP procedure. The animals were shocked or control-operated and euthanized at different times after surgery.

\section{Protocol design}

Sepsis was induced following a modification of a previously published method of CLP [10]. Briefly, animals were anesthetized with intraperitoneal injection of ketamine and xylazine (250 and $10 \mathrm{mg} / \mathrm{kg}$, respectively). After adequate anesthesia, the lower quadrants of the abdomen were shaved and the surgical area was disinfected. A longitudinal midline incision was made using a scalpel, and scissors were used 
to extend the incision into the peritoneal cavity. After intramuscular, fascial, and peritoneal incision, the cecum was located and exteriorized. In our experiments, the cecum was ligated at different lengths below the ileocecal valve to avoid bowel obstruction. Total cecal length was measured from the tip of the ascending cecum to the tip of the descending cecum. The cecum was then ligated at 5, 20, and $100 \%$ of its total length. For the "100\%" group, the cecum was ligated to the longest possible without bowel occlusion (Fig. 1).

The cecum was then perforated by a single puncture midway between the ligation and the tip of the cecum with a 20-G needle. We chose this needle diameter to obtain mid-grade lethal sepsis $[5,13,14]$. After removing the needle, a small amount of feces was extruded. The cecum was relocated, after which the fascia, abdominal musculature, and peritoneum were closed via simple running sutures; the skin was also sutured. The control mice were anesthetized and underwent laparotomy without puncture or cecal ligation and served as the control. The animals were shocked or control-operated and euthanized at different times depending on the set of experiments.

Immediately post-procedure, $1 \mathrm{ml}$ of saline was administered subcutaneously for fluid resuscitation (circa $0.045 \mathrm{ml} / \mathrm{g}$ ) [8, 14]. Pain control for CLP and sham mice was achieved with $0.05 \mathrm{mg} / \mathrm{kg}$ buprenorphine every $12 \mathrm{~h}$.

\section{Spontaneous mortality determination}

The first set of experiments consisted of observation of spontaneous mortality for each ligation length. The mice were then redistributed into subgroups of three to four in order to repeat the observation of mortality. The mortality was followed for 4 days after the CLP protocol.

\section{Renal function study and lactate dosage}

In the second set of experiments, control and CLP animals were euthanized at $6 \mathrm{~h}$.

We used serum lactates as a severity marker of septic state and serum creatinine concentration as a marker of renal dysfunction. Samples were collected $6 \mathrm{~h}$ after surgery by intracardiac puncture under general anesthesia (intraperitoneal injection of

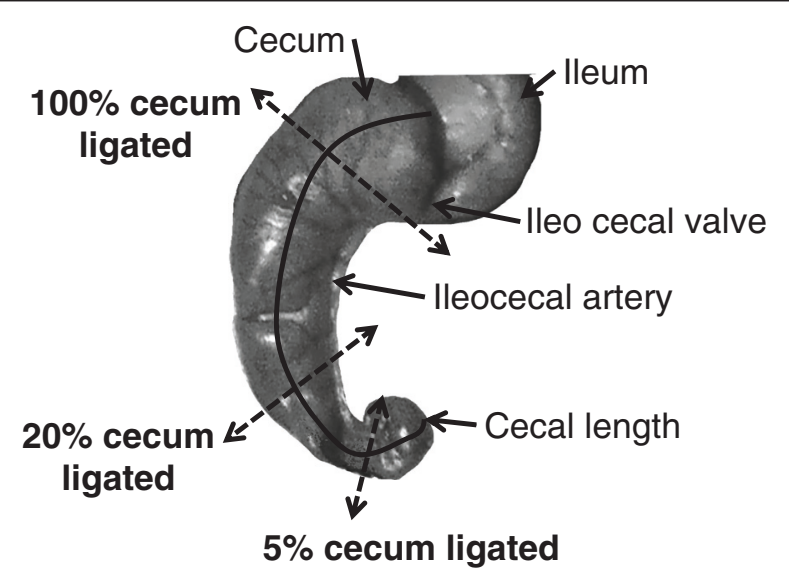

Fig. 1 Description of cecal length ligation methods. The total length of the cecum is represented by the full line. Dotted arrows are placed at each level of cecal ligation: 5, 20, and $100 \%$ of the total of cecum length 
$250 \mathrm{mg} / \mathrm{kg}$ ketamine and $10 \mathrm{mg} / \mathrm{kg}$ xylazine). Serum obtained after centrifugation was immediately frozen and stored at $-80{ }^{\circ} \mathrm{C}$ before being analyzed at the phenotyping platform (GenoToul Anexplo, Toulouse, France).

\section{Serum cytokines, bacterial blood culture, and leukocyte count}

In the third set of experiments, the mice were put down at 6 and $24 \mathrm{~h}$ for the control, " $5 \%$," "20\%," and "100\%," ligated groups. For the control and $20 \%$ ligated animals, we performed supplementary analysis at $48 \mathrm{~h}$.

We measured serum cytokines TNF- $\alpha$, IL-1 $\beta$, and IL-6. IL-10 concentrations were determined at 24 and $48 \mathrm{~h}$ for the control and $20 \%$ ligated groups. The samples were collected by intracardiac puncture under general anesthesia (intraperitoneal injection of $250 \mathrm{mg} / \mathrm{kg}$ ketamine and $10 \mathrm{mg} / \mathrm{kg}$ xylazine). The samples were then immediately frozen at $-80{ }^{\circ} \mathrm{C}$ and analyzed by Luminex technique (Bio-Rad Y60-00000YU Pro Mouse Cytokines Group 4-plex 1 x 96, Bio-Rad, Hercules, CA, USA) on the phenotyping platform (GenoToul Anexplo, Toulouse, France).

Leukocyte count was performed at the Phenotyping platform (GenoToul Anexplo, Toulouse, France) on the MICROS-60 hematology analyzer (Horiba ABX-Diagnostics, MA, USA). Datum is expressed in leukocytes per milliliter. Analysis was performed on $20-\mu \mathrm{l}$ samples of heparinized blood in the first hour following cardiac blood puncture.

Bacterial blood cultures were extracted for the $20 \%$ group $24 \mathrm{~h}$ after CLP. The samples were collected by cardiac puncture. Blood was serially diluted and cultured on a tryptic soy blood agar plate at $37^{\circ} \mathrm{C}$ for $48 \mathrm{~h}$ ( $n=10$ mice).

\section{Histological analysis}

A macroscopic examination was performed to look for abscess and pus collections in the peritoneal cavity at $24 \mathrm{~h}$ after surgery. Liver samples, preserved in $10 \%$ buffered formalin, were dehydrated and embedded in paraffin. Four-micrometer sections were stained with hematoxylin-eosin. The sections were then evaluated for signs of hypoperfusion and ischemic hepatitis or "shock liver."

\section{Mean arterial pressure measurement}

Mean arterial pressure (MAP) was measured under anesthesia in the "20 \%" ligated and control groups before surgery and at $24 \mathrm{~h}$ post-procedure. The femoral artery was catheterized. After surgery, a 5-min stabilization period was observed and femoral arterial blood pressure was monitored using a blood pressure analyzer (via a Statham P10 EZ transducer coupled to a TA 4000; Gould, Eichstetten, Germany) for $10 \mathrm{~min}$. The published results are the mean of MAP values measured every $30 \mathrm{~s}$.

\section{Statistics}

Values are not normally distributed and are expressed as median and range or interquartile range (IQR). To assess whether the measurements changed over time, Friedman's test was used. When Friedman's test was significant $(p<0.05)$, pair comparisons were performed using Wilcoxon's signed-rank test. Time comparison between groups was made using non-parametric Kruskal-Wallis test. When the Kruskal-Wallis test was significant $(p<0.05)$, then comparisons were made using 
the Dunn's post hoc test. Survival was analyzed by log-rank test. Analysis was performed using GraphPad Prism version 5.00 for Windows, GraphPad Software, La Jolla, CA USA, www.graphpad.com. Results with $p<0.05$ were considered statistically significant.

\section{Results}

\section{Mortality and organ dysfunctions are correlated with ligation length}

Median total cecal length was $29 \mathrm{~mm}(25-30 \mathrm{~mm})$. For the ligated groups, measurements of ligated ceca were the following: $2 \mathrm{~mm}(1-2 \mathrm{~mm})$ for the $5 \%$ ligated group, $6 \mathrm{~mm}(5-7 \mathrm{~mm})$ for the $20 \%$ ligated group, and $21 \mathrm{~mm}(19-25 \mathrm{~mm})$ for the $100 \%$ ligated group. Mortality was evaluated at different ligation lengths of standardized CLP by simple puncture with a 20-G needle. Our results indicate that the ligation length influences mortality (Fig. 2). At the end of the 96-h follow-up period, we observed $100 \%$ mortality in the $100 \%$ ligated group, $88 \%$ mortality in the $20 \%$ ligated group, and $20 \%$ mortality in the $5 \%$ ligated group $(p<0.001)$. The median survival time was $24 \mathrm{~h}$ for the $100 \%$ ligated group and $44 \mathrm{~h}$ for the $20 \%$ group. The median survival time of the $5 \%$ ligated group could not be determined because of the low number of deaths at the end of the observation period ( $80 \%$ of survival). Because of death rapidity in the $100 \%$ group, we macroscopically analyzed ceca $24 \mathrm{~h}$ post-procedure when the animals were put down for serum collection. The mice with cecum ligated at $100 \%$ presented with ischemia of the ligated component in contrast to other groups (Fig. 3c). Animals in the $20 \%$ group developed macroscopic cecal abscesses (Fig. 3a, b).

Serum creatinine and blood lactate also varied with ligation distance. Blood lactate increased proportionally with the length of ligated cecum (Fig. 4a). For the $5 \%$ ligated group, it did not differ from the control group. For the $20 \%$ and $100 \%$ ligated groups, blood lactate increased up to $2.6 \mathrm{mmol} / \mathrm{l}(1.2-4.2)$ for the $20 \%$ group and up to $3.2 \mathrm{mmol} / \mathrm{l}(1.2-5.7)$ for the $100 \%$ group. At $6 \mathrm{~h}$, serum creatinine increased by 1.5 -

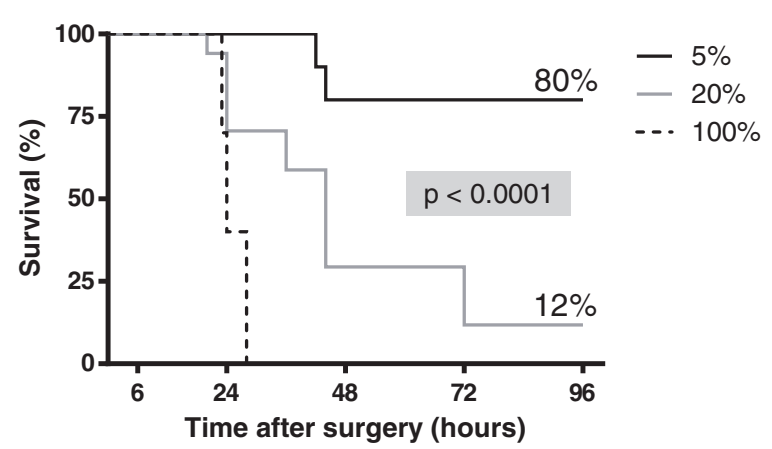

Fig. 2 Survival following ligation of $5 \%(n=10), 20 \%(n=17)$, and $100 \%(n=10)$ of total cecal length within $96 \mathrm{~h}$ after CLP procedure. At $24 \mathrm{~h}$, survival was $100 \%$ for the $5 \%$ group, $70 \%$ for $20 \%$ group, and $40 \%$ for $100 \%$ group (log-rank test, $p<0.05$ ). At $96 \mathrm{~h}$, we observed $100 \%$ mortality in the $100 \%$ ligated group, $88 \%$ mortality in the $20 \%$ ligated group, and $20 \%$ mortality in the $5 \%$ ligated group (log-rank test, $p<0.0001$ ). The median survival time was $24 \mathrm{~h}$ for the $100 \%$ ligated group and $44 \mathrm{~h}$ for the $20 \%$ group. The median survival of the $5 \%$ ligated group could not be determined because of the low number of deaths at the end of the observation period ( $80 \%$ of survival) 

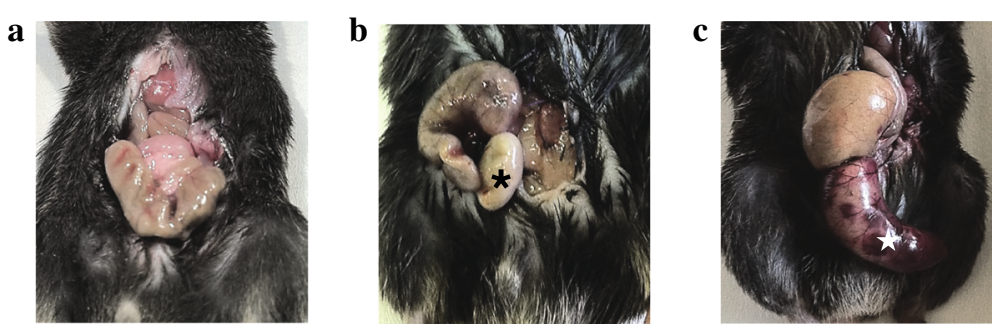

Fig. 3 Macroscopic aspects of ceca $24 \mathrm{~h}$ after CLP procedure. a Sham-operated mouse. b Mouse with $20 \%$ of cecum ligated; we observed cecal abscess (**). c Mouse with cecum ligated at $100 \%$ presented an ischemia of the ligated part

fold in the $100 \%$ group compared to the control mice: $26.2 \mu \mathrm{mol} / \mathrm{l}(17.4-73.8)$ vs $15.2 \mu \mathrm{mol} / \mathrm{l}(6.6-23.9)$. In the $20 \%$ group, serum creatinine was higher at $16.9 \mu \mathrm{mol} / \mathrm{l}$ (12.5-39) when compared to the control group (Fig. 4b).

\section{Inflammatory status differs in accordance with ligation length}

Inflammatory response to CLP length was evaluated by serum cytokines. TNF- $\alpha$, IL-1 $\beta$, and IL- 6 concentrations were measured at 6 and $24 \mathrm{~h}$ for all the groups. We calculated an IL-6/IL-10 ratio at $24 \mathrm{~h}$ to determine the balance between pro- and antiinflammatory responses. At $24 \mathrm{~h}$ post-procedure, only four animals in the $100 \%$ group were still alive.

At $6 \mathrm{~h}$ after the procedure, pro-inflammatory cytokines were significantly increased in the ligated groups compared to the control group. TNF- $\alpha$ was increased in the $5 \%$ group (212.4 pg/ml (56.7-313.7)), $20 \%$ (187.6 pg/ml (105.6-317)), and $100 \%$ group (190.9 pg/ $\mathrm{ml}(112.1-317.1))$ compared to the control group (136.7 pg/ml (50.3-181); $p<0.05)$. We did not observe any difference in amongst the ligated groups (Fig. 5a). At 24 h, TNF- $\alpha$ quickly decreased or animals subsequently died. There was no difference between the $100 \%$ groups because of the few number of survivors at $24 \mathrm{~h}$ (less than five) (Fig. $5 \mathrm{a}$ ).

IL-1 $\beta$ significantly increased at $6 \mathrm{~h}$ for the groups $5 \%(218.8 \mathrm{pg} / \mathrm{ml}(26-320.8))$ and $20 \%(213.4 \mathrm{pg} / \mathrm{ml}(119-388.6))$ compared to the control group (93.5 pg/ml (67.5-

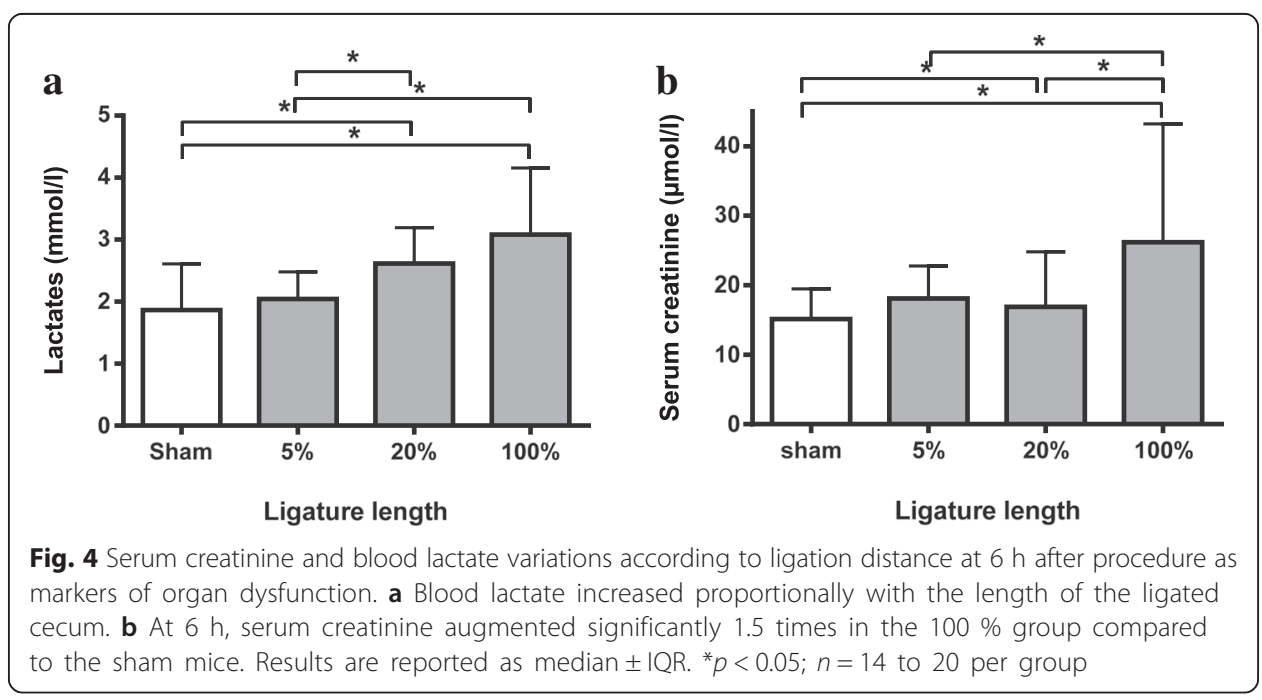



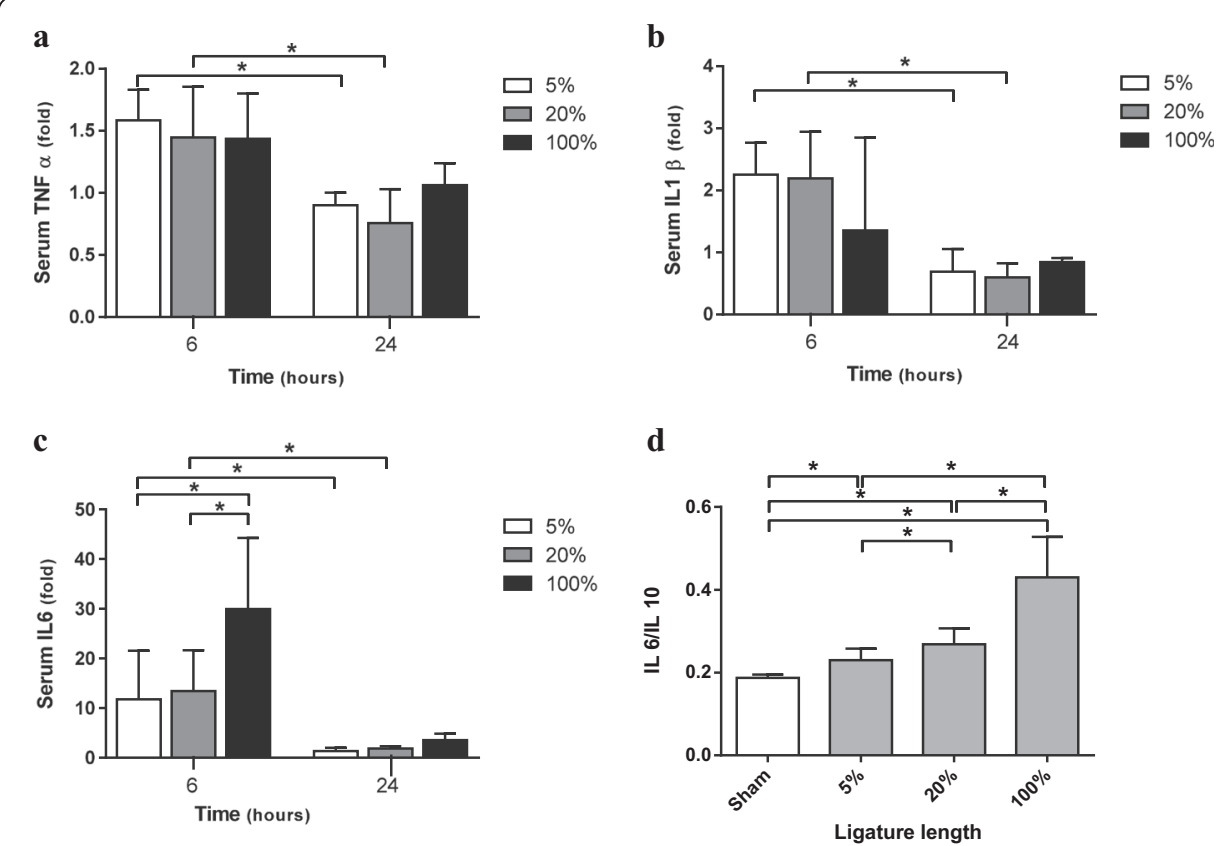

Fig. 5 Inflammatory response to CLP length evaluated by serum cytokines. TNF- $a$, IL-1 1 , and IL- 6 concentrations were measured for all the groups at $6 \mathrm{~h}(n=10$ for sham-operated and each length of the ligated cecum) and $24 \mathrm{~h}$ ( $n=10$ for the sham-operated, $5 \%$, and $20 \% ; n=4$ for the $100 \%$ ligated group). At $24 \mathrm{~h}$ after procedure, only $40 \%$ of the animals in the $100 \%$ group were alive. We expressed serum TNF-a, IL-1 $\beta$, and IL-6 concentrations as fold increases relative to time-matched sham laparotomy (a-c). a Serum TNF-a concentrations. We did not observe any difference between the ligated groups at $\mathrm{H} 6$ or $\mathrm{H} 24$, but a decrease in time. $\mathbf{b}$ Serum IL-1 $\beta$ concentrations. We observed a decrease in time, but no difference between the ligated groups at the same time. c Serum IL-6 concentrations. Besides decrease in time, we observed significant higher levels of serum IL-6 at H6 in the $100 \%$ compared to the $5 \%$ and $20 \%$ ligated groups. d Serum IL-6/L-10 ratio. The longer the ligated cecum was, the more the imbalance was significantly in favor of pro-inflammatory response. Results are reported as median $\pm \mathrm{IQR} ;{ }^{*} p<0.05$

$150.1) ; p<0.05)$. On the other hand, IL-1 $\beta$ serum concentrations of the $100 \%$ ligated group were not different from the control group (128.4 pg/ml (60.8-354.9)). IL-1 $\beta$ concentrations did not increase in accordance with the length of ligation at $6 \mathrm{~h}$ but decreased at $24 \mathrm{~h}$ nevertheless (Fig. 5b).

At 6 h, IL-6 serum concentrations of ligated groups were at least 15 times higher than in the control group (Fig. 5 c; $p<0.05$ ). Serum IL-6 concentrations reached $1916 \mathrm{pg} / \mathrm{ml}$ (961-4141) for the $20 \%$ ligated group and were evidently increased for the $100 \%$ group, with a median value of $4262 \mathrm{pg} / \mathrm{ml}$ (2070-7723). Like other proinflammatory cytokines, IL-6 concentrations decreased at $24 \mathrm{~h}$ (Fig. 5c).

At $24 \mathrm{~h}$, when observing the pro- and anti-inflammatory balance (IL-6/IL-10), the longer the ligated cecum, the more significant the pro-inflammatory status was (Fig. 5d).

\section{The "20\%" ligated group presented all characteristics of septic shock}

With these results, we more closely monitored the $20 \%$ ligated group. At $24 \mathrm{~h}$ after surgery, without any resuscitation the animals presented with a decreased MAP below $65 \mathrm{mmHg}$ compared to the control mice (Fig. 6a). 


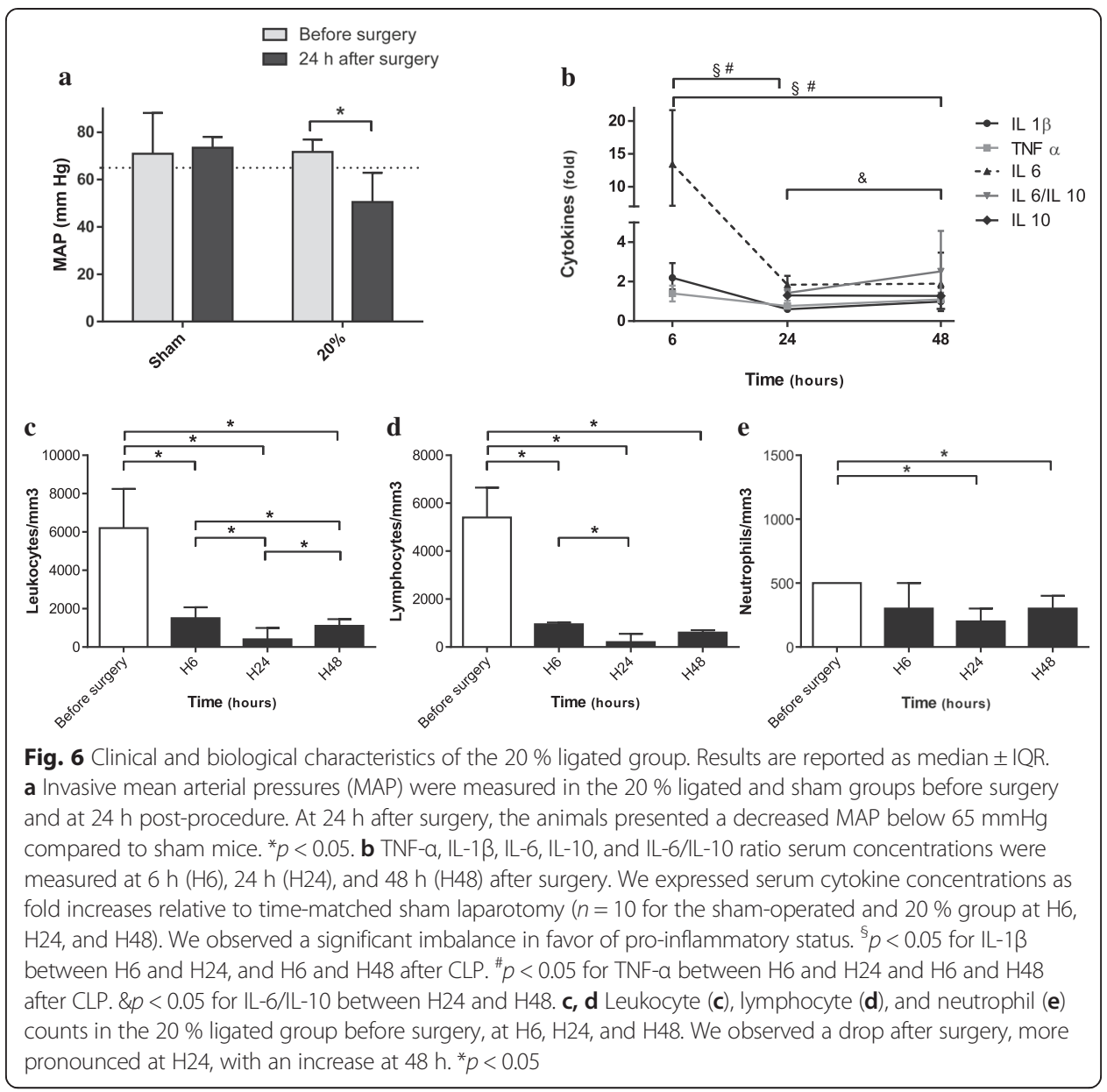

This hypotension was associated with sepsis in the $20 \%$ ligated group. We observed cecal abscesses when compared to control mice (Fig. 7a, b), and blood cultures at 24-h post-procedure were positive with enteric bacteria such Citrobacter braakii and Enterococcus faecalis (40 \%).

This was associated with a drop in leukocyte count due to leucopenia, which peaked at $24 \mathrm{~h}$ (Fig. 6c, d, e). At 48 h, leukocyte count increased but was diminished compared to time before surgery. The cytokine profile was pro-inflammatory, including status at $48 \mathrm{~h}$. IL-6 levels remained high in survivors at 2.9 compared to those in the control and were not counterbalanced by IL-10 levels. At 48 h, IL-6/ IL-10 ratio increased compared to $24 \mathrm{~h}$ after CLP, what highlights the persistence of inflammation (Fig. 6c).

When observing macroscopic liver morphology, we found a patchy appearance corresponding to pale ischemic areas in contrast to the control liver (Fig. 7c, d). These areas displayed centrilobular necrosis of hepatocytes (Fig. 7f).

\section{Discussion}

While the CLP model is the most widely used model for experimental sepsis, the length of cecal ligation is often inaccurately described. Our results underline that, in mice, length of cecal ligation is a major determinant of mortality and sepsis severity. Organ 

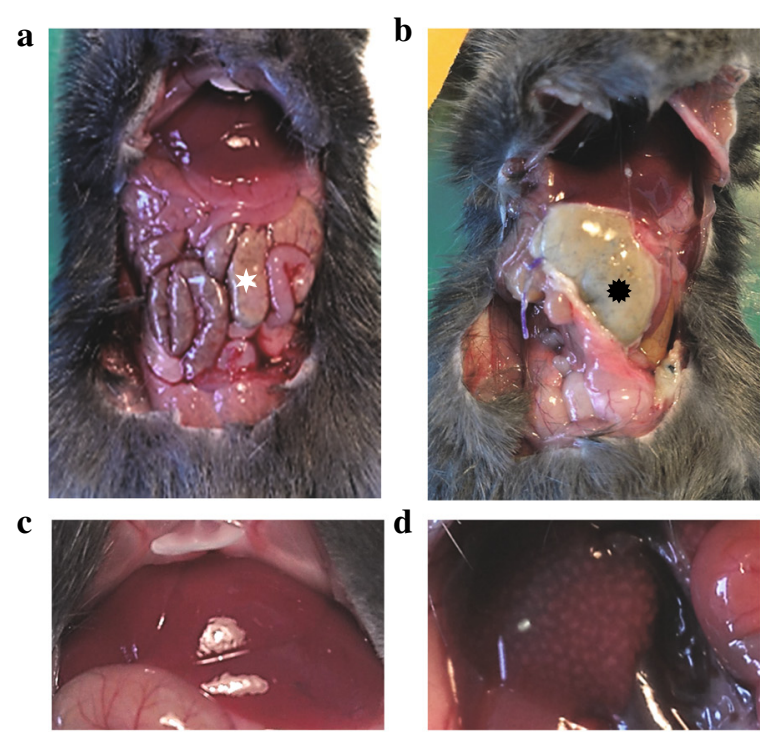

$\mathbf{e}$
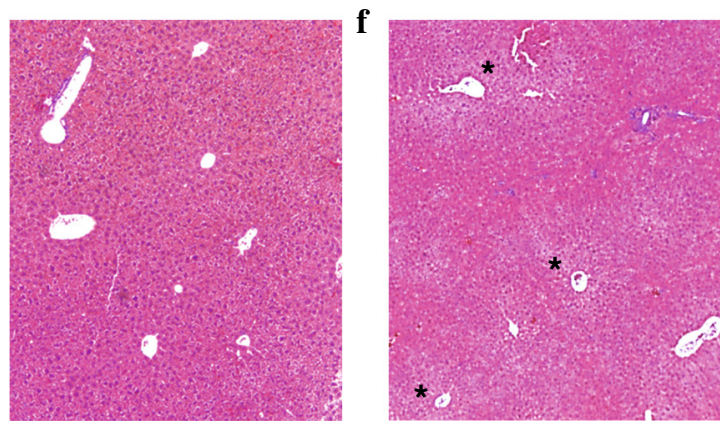

Fig. 7 Aspect of peritoneal cavity of the sham-operated mice $(\mathbf{a}, \mathbf{c}, \mathbf{e})$ and the $20 \%$ ligated mice $(\mathbf{b}, \mathbf{d}, \mathbf{f})$ $24 \mathrm{~h}$ after surgery. $\mathbf{a}$ The cecum is identified by a white star. $\mathbf{b}$ The $20 \%$ ligated animal with cecal abscess

When observing liver macroscopic morphology, we found a patchy appearance corresponding to pale ischemic areas (d) in contrast to the sham liver (c). $\mathbf{f}$ These areas were centrilobular necrosis of hepatocytes (asterisk) (e and $\mathbf{f}$ hematoxylin-eosin coloration $\times 50$ )

dysfunction markers and pro-inflammatory status increased with ligation length. The "20 \%" ligated group presented all characteristics of septic shock with a delayed mortality compared to the "100 \%" group, allowing further studies as to the effect of different treatments or physiopathology. In the " $20 \%$ " group, we confirmed the presence of sepsis associated with hypotension below $65 \mathrm{mmHg}$, pro-inflammatory balance with high IL-6 levels and augmented IL-6/IL-10 ratio, organ dysfunction, hyperlactatemia, elevated serum creatinine, and hepatocyte centrilobular necrosis.

Length of ligated cecum influenced mortality in our mice model with high lethality in the $20 \%$ and $100 \%$ groups (at 4 days, 88 and $100 \%$ mortality, respectively). As we found in our results, the percentage of cecum ligation is more accurate than a standard length (as length can vary from 25 to $30 \mathrm{~mm}$ ). Rittirsch briefly described this influence in his mouse CLP procedure but did not characterize the model except for survival rate and for pro-inflammatory cytokines in his mid-grade sepsis group [10, 12]. Singleton showed the same influence more extensively but in a rat CLP model [15]. In his model, at $96 \mathrm{~h}$ a ligation length of $20 \%$ allowed a $60 \%$ survival rate whereas " $25 \%$ " of a ligated cecum caused higher mortality with only a $24 \%$ survival rate. Our data confirm the importance of this variable in mouse CLP model. Singleton's results could not be 
transposed a priori because of differences in anatomy and vascularization of these rodents' ceca. In fact, the same proportion of ligated cecum between these two breeds of animals does not exactly have same consequence in terms of lethality. Our study was not designed to determine which mechanisms were involved in this phenomenon. However, we suppose that mortality in the $100 \%$ ligated group is related to intestinal ischemia rather than sepsis. We observed macroscopic cecal necrosis early after procedure. As discussed in Singleton's study concerning rats, the response amplitude to cecum ligated length may be due to the amount of feces stored in the ligated portion and thus create bacterial inoculum that may translocate or be locally pathogenic (e.g., by forming abscesses like in the $20 \%$ group).

Inflammatory response mediated by cytokines plays a major role in sepsis evolution $[16,17]$. Pro-inflammatory cytokines like TNF- $\alpha$, IL-1 $\beta$, and IL-6 are responsible for severe manifestations in sepsis and septic shock [17, 18]. In our model, apart from IL-6 measurements, differences between groups for other cytokines are not clinically relevant when considering the same measurement time. Serum TNF$\alpha$ levels peak at $120 \mathrm{~min}$ in mouse CLP models [19]. It is the first cytokine produced in response to aggression, and it promotes the activation of immune cells and the release of immunoregulatory mediators [20]. In our model, TNF- $\alpha$ was significantly increased at $6 \mathrm{~h}$ in ligated groups compared to control mice. However, serum TNF- $\alpha$ concentrations were not proportional to ligation length as described previously in rats [15]. We detected serum TNF- $\alpha$ in the control mice as well and did not find any differences between the ligated groups; this finding was opposite to Singleton et al.'s in rats [15]. These findings are in accordance with other studies observing severity in mice CLP models. Serum TNF- $\alpha$ levels did not differ between the more severe groups which were defined by the puncture size [14]. One explanation may be the difference in pro-inflammatory response influenced by genetic background. Serum TNF- $\alpha$ profiles over time are different after CLP between $\mathrm{A} / \mathrm{J}$ and $\mathrm{C} 57 \mathrm{BL} / 6 \mathrm{~J}$ mice; therefore, we infer it could be dissimilar between rats and mice [21]. Moreover, TNF- $\alpha$ neutralization does not improve survival in mouse CLP model [22-24]. This underlines that serum TNF- $\alpha$ levels are not necessarily linked to severity of insult in this model.

Serum IL-1 $\beta$ levels were higher in the " $5 \%$ " and " $20 \%$ " groups at $6 \mathrm{~h}$ compared to the levels at $24 \mathrm{~h}$. We did not observe any difference between groups at the same time or between 6 and $24 \mathrm{~h}$ for the "100 \%" ligated group. There is little data in the literature concerning IL-1 $\beta$ in mouse CLP modeling. Initial descriptions of the inflammatory profile in this mouse model did not detect serum IL-1 $\beta$ [8]. In the mouse CLP model, this cytokine seemed to be significantly elevated in the sera of animals that died before the fifth day post-procedure [25]. In a study evaluating needle size in CLP, only the group with the largest puncture presented with a significant elevation in serum Il-1 $\beta$ at $24 \mathrm{~h}$ [13]. In our case, the lack of difference between groups may be explained by the regulation of IL- $1 \beta$ secretion and the small number of surviving animals in the $100 \%$ group at $24 \mathrm{~h}[26]$.

Concerning serum IL-6, our model is in accordance with previous results indicating that serum IL-6 levels increase proportionally with mortality at $6 \mathrm{~h}$ after mouse CLP $[27,28]$. As described in the literature, the $20 \%$ and $100 \%$ groups, which presented with the highest mortality rates, had serum IL-6 concentrations near or superior to 
$2000 \mathrm{pg} / \mathrm{ml}$. This breakpoint predicts mortality within 3 days with a specificity of $97 \%$ and sensitivity of $58 \%$ [27]. When observing the pro- and anti-inflammatory balances, the IL-6/IL-10 ratio was higher in groups with larger lengths of ligated cecum. This ratio has been shown to be predictive of the outcome of patients with systemic inflammatory response syndrome [29-31].

We use in our model low dose of buprenorphine $(0.05 \mathrm{mg} / \mathrm{kg}$ every $12 \mathrm{~h})$ to achieve analgesia as stated by the Office of Laboratory Animal Welfare [32]. Morphine is known to increase pro-inflammatory mediators; however, low dose of buprenorphine seems to have no effect on mortality and inflammatory response $[33,34]$. Moreover, we gave this analgesic drug both to sham and CLP mice; hence, we believe that buprenorphine is not a cofounding factor in our model.

In our study, we focused on the $20 \%$ ligatured group. This was done since we chose this group for further experimentation and wanted to ensure that the results were consistent. Furthermore, this group corresponds to a septic shock group. The animals of this group presented both clinical and biological elements of septic shock. They were hypotensive with a MAP below $65 \mathrm{mmHg}$. The cytokine profile was in favor of a pro-inflammatory imbalance with markers predictive of mortality, such as IL-6 and the IL-6/IL-10 ratio [27, 30]. At 48 h, IL-6/IL-10 ratio increased compared to that at $24 \mathrm{~h}$ after CLP, which shows the persistence of inflammation. Moreover, this group presented with a drop in leucocyte count secondary to lymphopenia, which decreased even further at $24 \mathrm{~h}$. Other studies described this change in a complete blood count, with the same kinetics $[8,13,35$, 36]. The lymphopenia was secondary to sepsis-induced apoptosis and is correlated to the severity of an immunosuppressive phase and its late complications [37, 38].

We acknowledge that our study has potential limitations. First, we did not perform the same analysis in the "100 \%" group as we did in the "20\%" group because of the high lethality rate. We chose animals with the same genetic background, age, and gender to limit experimental variability secondary to differences in inflammatory response and maturity of the immune system [11,39]. Because of the mice's age, we did not have enough animals to compensate for the mortality of the $100 \%$ ligated group. Furthermore, our study lacked evaluation of anti-inflammatory balance within the first hours post-procedure. As previous studies on mouse CLP modeling described late IL-10 serum elevation, we chose to measure levels at 24 and $48 \mathrm{~h}[13,14]$. We were not able to determine if the $100 \%$ group had very early pro- and anti-inflammatory imbalance, which has been shown to be predictive of mortality [25]. Third, we are aware that we have not analyzed exhaustively the cytokine response to cecal ligation. For example, other cytokines such as IL-12 or interferon- $\gamma$ play a central role in septic inflammatory response [40].

\section{Conclusions}

Our study suggests that the length of cecal ligation is a major severity factor in the mouse CLP model when needle size and the number of punctures are controlled. Furthermore, it underlines differences in the inflammatory response between rats and mice. Therefore, this experimental model should be performed with high consistency in future protocol designs. In order to accurately compare studies, ligature length used in protocols should be described. 


\section{Acknowledgements}

This work should be attributed to the Department of Anesthesia and Intensive Care. Support was provided solely by institutional and department sources. We acknowledge Maria Ahmad for her kind help in the English editing.

\section{Authors' contributions}

SR conceived and designed the experiments. SR, FV-B, VM-D, and MB performed the experiments. SR, VM-D, and JMC analyzed the data. SR, FV-B, VM, IT, and OF wrote the paper. All authors read and approved the final manuscript.

\section{Competing interests}

The authors declare that they have no competing interests.

\section{Ethics approval and consent to participate}

Animal experimentation was performed according to national and institutional animal care and ethical guidelines and was approved by the local board.

\section{Author details}

${ }^{1}$ Department of Anesthesiology and Intensive Care, Rangueil Hospital, University Hospital of Toulouse, 1 Avenue du Professeur Jean Poulhès TSA 50032, 31059 Toulouse, Cedex 9, France. ${ }^{2}$ Inserm/UPS UMR 1048 - I2MC, Equipe 3, Toulouse, France. ${ }^{3} E A 4564$ - MATN - Laboratoire de Modélisation de I'Agression Tissulaire et de la Nociception Toulouse, Institut Louis Bugnard (IFR 150), Toulouse, France. ${ }^{4}$ Inserm/UPS UMR 1048 - I2MC, Equipe 12, Toulouse, France. ${ }^{5}$ Department of Physiology, Rangueil Hospital, University Hospital of Toulouse, 1 Avenue du Professeur Jean Poulhès TSA 50032, 31059 Toulouse Cedex 9, France.

Received: 17 February 2016 Accepted: 7 July 2016

Published online: 18 July 2016

\section{References}

1. Hotchkiss RS, Karl IE (2003) The pathophysiology and treatment of sepsis. N Engl J Med 348:138-150

2. Dejager L, Pinheiro I, Dejonckheere E, Libert C (2011) Cecal ligation and puncture: the gold standard model for polymicrobial sepsis? Trends Microbiol 19:198-208

3. Roquilly A, Gautreau L, Segain JP, de Coppet P, Sebille V, Jacqueline C, Caillon J, Potel G, Lejus C, Josien R, Asehnoune K (2010) CpG-ODN and MPLA prevent mortality in a murine model of post-hemorrhageStaphylococcus aureus pneumonia. PLoS One 5, e13228

4. Baker CC, Chaudry $\mathrm{H}$, Gaines HO, Baue AE (1983) Evaluation of factors affecting mortality rate after sepsis in a murine cecal ligation and puncture model. Surgery 94:331-335

5. Hubbard WJ, Choudhry M, Schwacha MG, Kerby JD, Rue LW III, Bland Kl, Chaudry IH (2005) Cecal ligation and puncture. Shock 24(1):52-57

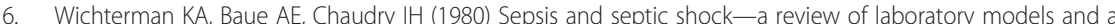
proposal. J Surg Res 29:189-201

7. Deitch EA (1998) Animal models of sepsis and shock: a review and lessons learned. Shock 9:1-11

8. Remick DG, Newcomb DE, Bolgos GL, Call DR (2000) Comparison of the mortality and inflammatory response of two models of sepsis: lipopolysaccharide vs. cecal ligation and puncture. Shock 13:110-116

9. Freise H, Bruckner UB, Spiegel HU (2001) Animal models of sepsis. J Invest Surg 14:195-212

10. Rittirsch D, Huber-Lang MS, Flierl MA, Ward PA (2009) Immunodesign of experimental sepsis by cecal ligation and puncture. Nat Protoc 4:31-36

11. Deitch EA (2005) Rodent models of intra-abdominal infection. Shock 24(Suppl 1):19-23

12. Rittirsch D, Flierl MA, Nadeau BA, Day DE, Huber-Lang M, Mackay CR, Zetoune FS, Gerard NP, Cianflone K, Kohl J, Gerard C, Sarma JV, Ward PA (2008) Functional roles for C5a receptors in sepsis. Nat Med 14:551-557

13. Ebong S, Call D, Nemzek J, Bolgos G, Newcomb D, Remick D (1999) Immunopathologic alterations in murine models of sepsis of increasing severity. Infect Immun 67:6603-6610

14. Walley KR, Lukacs NW, Standiford TJ, Strieter RM, Kunkel SL (1996) Balance of inflammatory cytokines related to severity and mortality of murine sepsis. Infect Immun 64:4733-4738

15. Singleton KD, Wischmeyer PE (2003) Distance of cecum ligated influences mortality, tumor necrosis factor-alpha and interleukin-6 expression following cecal ligation and puncture in the rat. Eur Surg Res 35:486-491

16. Reddy RC, Chen GH, Tekchandani PK, Standiford TJ (2001) Sepsis-induced immunosuppression: from bad to worse. Immunol Res 24:273-287

17. Wheeler AP, Bernard GR (1999) Treating patients with severe sepsis. N Engl J Med 340:207-214

18. Cinel I, Opal SM (2009) Molecular biology of inflammation and sepsis: a primer. Crit Care Med 37:291-304

19. Villa P, Sartor G, Angelini M, Sironi M, Conni M, Gnocchi P, Isetta AM, Grau G, Buurman W, van Tits LJ et al (1995) Pattern of cytokines and pharmacomodulation in sepsis induced by cecal ligation and puncture compared with that induced by endotoxin. Clin Diagn Lab Immunol 2:549-553

20. de Jong HK, van der Poll T, Wiersinga WJ (2010) The systemic pro-inflammatory response in sepsis. Journal of innate immunity 2:422-430

21. Torres MB, De Maio A (2005) An exaggerated inflammatory response after CLP correlates with a negative outcome. J Surg Res 125:88-93

22. Newham P, Ross D, Ceuppens P, Das S, Yates JW, Betts C, Reens J, Randall KJ, Knight R, McKay JS (2014) Determination of the safety and efficacy of therapeutic neutralization of tumor necrosis factor-alpha (TNF-alpha) using AZD9773, an anti-TNF-alpha immune Fab, in murine CLP sepsis. Inflamm Res 63:149-160

23. Eskandari MK, Bolgos G, Miller C, Nguyen DT, DeForge LE, Remick DG (1992) Anti-tumor necrosis factor antibody therapy fails to prevent lethality after cecal ligation and puncture or endotoxemia. J Immunol 148:2724-2730 
24. Lorente JA, Marshall JC (2005) Neutralization of tumor necrosis factor in preclinical models of sepsis. Shock 24(Suppl 1):107-119

25. Osuchowski MF, Welch K, Siddiqui J, Remick DG (2006) Circulating cytokine/inhibitor profiles reshape the understanding of the SIRS/CARS continuum in sepsis and predict mortality. J Immunol 177:1967-1974

26. Dinarello CA (2005) Interleukin-1 beta. Crit Care Med 33:S460-\$462

27. Remick DG, Bolgos GR, Siddiqui J, Shin J, Nemzek JA (2002) Six at six: interleukin-6 measured $6 \mathrm{~h}$ after the initiation of sepsis predicts mortality over 3 days. Shock 17:463-467

28. Song M, Kellum JA (2005) Interleukin-6. Crit Care Med 33:S463-S465

29. Taniguchi T, Koido Y, Aiboshi J, Yamashita T, Suzaki S, Kurokawa A (1999) The ratio of interleukin-6 to interleukin10 correlates with severity in patients with chest and abdominal trauma. Am J Emerg Med 17:548-551

30. Taniguchi T, Koido Y, Aiboshi J, Yamashita T, Suzaki S, Kurokawa A (1999) Change in the ratio of interleukin-6 to interleukin-10 predicts a poor outcome in patients with systemic inflammatory response syndrome. Crit Care Med 27:1262-1264

31. Weis F, Beiras-Fernandez A, Schelling G, Briegel J, Lang P, Hauer D, Kreth S, Kaufmann I, Lamm P, Kilger E (2009) Stress doses of hydrocortisone in high-risk patients undergoing cardiac surgery: effects on interleukin-6 to interleukin-10 ratio and early outcome. Crit Care Med 37:1685-1690

32. Welfare OoLA (2015) Public Health Service policy on humane care and use of laboratory animals. In: Editor (ed)^(eds) Book Public Health Service policy on humane care and use of laboratory animals.

33. Cotroneo TM, Hugunin KM, Shuster KA, Hwang HJ, Kakaraparthi BN, Nemzek-Hamlin JA (2012) Effects of buprenorphine on a cecal ligation and puncture model in C57BL/6 mice. J Am Assoc Lab Anim Sci 51:357-365

34. Odunayo A, Dodam JR, Kerl ME, DeClue AE (2010) Immunomodulatory effects of opioids. J Vet Emerg Crit Care 20:376-385

35. Ebong SJ, Call DR, Bolgos G, Newcomb DE, Granger Jl, O'Reilly M, Remick DG (1999) Immunopathologic responses to non-lethal sepsis. Shock 12:118-126

36. Muenzer JT, Davis CG, Chang K, Schmidt RE, Dunne WM, Coopersmith CM, Hotchkiss RS (2010) Characterization and modulation of the immunosuppressive phase of sepsis. Infect Immun 78:1582-1592

37. Hotchkiss RS, Tinsley KW, Swanson PE, Chang KC, Cobb JP, Buchman TG, Korsmeyer SJ, Karl IE (1999) Prevention of lymphocyte cell death in sepsis improves survival in mice. Proc Natl Acad Sci U S A 96:14541-14546

38. Hotchkiss RS, Monneret G, Payen D (2013) Sepsis-induced immunosuppression: from cellular dysfunctions to immunotherapy. Nat Rev Immunol 13:862-874

39. Turnbull IR, Wlzorek JJ, Osborne D, Hotchkiss RS, Coopersmith CM, Buchman TG (2003) Effects of age on mortality and antibiotic efficacy in cecal ligation and puncture. Shock 19:310-313

40. Wiersinga WJ (2011) Current insights in sepsis: from pathogenesis to new treatment targets. Curr Opin Crit Care $17: 480-486$

\section{Submit your manuscript to a SpringerOpen ${ }^{\circ}$} journal and benefit from:

- Convenient online submission

- Rigorous peer review

- Immediate publication on acceptance

- Open access: articles freely available online

- High visibility within the field

- Retaining the copyright to your article 